\title{
CONFORMATIONAL BEHAVIOUR OF POLY(2,6-DIMETHYL-1,4-PHENYLENE OXIDE) IN SOLUTION--I
}

\section{INTRINSIC VISCOSITY AS A FUNCTION OF TEMPERATURE}

\author{
J. W. A. van den Berg, G. van de Ridder and C. A. Smolders \\ Twente University of Technology, Department of Chemical Technology, \\ P.O. Box 217, 7500 AE Enschede, The Netherlands
}

(Received 21 November 1980; in revised form 3 March 1981)

\begin{abstract}
Intrinsic viscosities [ $\eta$ ] of poly(2,6-dimethyl-1,4-phenylene oxide) (PPO) solutions have been measured as a function of temperature between 60 and $25^{\circ} \mathrm{C}$. The solvents were toluene and trichloroethene. In both solvents, the $[\eta]-T$ curve exhibited a point of inflection in the range $45-35^{\circ}$. This phenomenon is explained as a conformational transition, which is possibly involved in the nucleation process of the solution crystallization of PPO. Assuming constancy of the coil expansion factor $\alpha_{1}$ and the solvent draining over the whole temperature interval, a slight increase of characteristic parameter $C_{x}$ with decreasing temperature has been calculated.
\end{abstract}

\section{INTRODUCTION}

Dilute solutions (say $<5-10 \%$ ) of poly(2,6-dimethyl-1,4-phenylene oxide) (PPO) in toluene show phase separation phenomena upon cooling to appropriate temperatures. According to Koenhen et al. [1,2] these phenomena may be interpreted consistently as crystallization phenomena. Turska and Janeczek [3] found that PPO may also crystallize from decalin. Data of Broens et al. [4] suggest that trichloroethene (TCE) should be "better" than toluene as a solvent for PPO: solutions of PPO in TCE must be cooled to much lower temperatures than solutions in toluene in order to obtain phase separation. An alternative way to obtain crystallization is to add a $n$-alcohol to the PPO-TCE mixtures [4]

Koenhen et al. [2] performed kinetic studies on self-seeded and on homogenous crystallizations of PPO from dilute toluene solutions. They inferred that the crystallization process should involve a nucleation process in two steps, the first being a conformational transition, the second nucleation and growth of crystallites.

In this paper we report the results of measurements concerning the supposed first step of the nucleation process, viz. the conformational transition. We chose as experimental methods light scattering and viscometry as a function of temperature $\left(25-60^{\circ} \mathrm{C}\right)$, as many conformational transitions have been detected in this way [5-9]. As already stated, PPO does not crystallize as readily from TCE as from toluene. The conformational transition, supposed to be related to the incipience of nucleation, should therefore occur in TCE solutions in a lower temperature region than in toluene solutions.

Our study differs from earlier studies $[10,11]$ on the conformation of PPO; the main difference is that we measured at temperatures in the vicinity of the melting point curve of the PPO-toluene system (cf. Ref. [1], Fig. 6) in the dilute region, whereas others did not study the temperature dependence of conformation [10], or measured only at 25 and $90^{\circ} \mathrm{C}$ [11].

These earlier studies showed PPO molecules to be random coils, with unperturbed dimensions close to those of a freely rotating chain. In this respect, they may be said to exhibit "flexibility". This "dimensional" or "equilibrium" flexibility should be clearly distinguished however from the "kinetic" (or "dynamic") flexibility, which for PPO is rather low. Thus, PPO molecules will be referred to as "tightly coiled" (cf. Akers et al. [11]), or "random coils with low chain mobility", or "stiff coils".

\section{EXPERIMENTAL}

\section{Polymer samples}

We prepared several samples of PPO according to the recipe of McCaffery [12]. The highest mol wt sample had $\overline{\mathbf{M}}_{n} 33,000, \overline{\mathrm{M}}_{\mathrm{w}} 58,000$ and $\overline{\mathbf{M}}_{\mathrm{z}}$ 96,000. Fractionations according to published procedures $[10,11]$ were unsuccessful, giving only small yields, with $\overline{\mathrm{M}}_{w}$ never above 100,000 . Hence we adopted the following procedure.

$100 \mathrm{~g}$ of PPO freshly prepared was dissolved in 11 . of boiling toluene. The solution was cooled and kept for $24 \mathrm{hr}$ at $27^{\circ} \mathrm{C}$ in a thermostat. (During this time the solution was constantly stirred.) The stirrer was then stopped. to allow the crystallized PPO to settle for another $24 \mathrm{hr}$. The slurry containing finely divided PPO was filtered on a glass filter No. 4 and dried in vacuo at $80^{\circ} \mathrm{C}$. The dried product was redissolved to give a $10 \%$ solution in chloroform and precipitated by dripping the solution with stirring in a fivefold excess of methanol. The voluminous flocculate was filtered through a Buchner funnel, washed several times with methanol and dried as above

As a result of this procedure, we obtained from every synthesized PPO sample one fraction of a slightly higher $\overline{\mathrm{M}}_{\mathrm{w}}$. Thus, from $100 \mathrm{~g}$ of the highest mol wt sample we were able to synthesize (see above), we obtained $15 \mathrm{~g}$ of the fraction called PPO-9 in Table 1.

\section{Solvents and solutions}

Toluene (analytical grade, Merck) and TCE (stabilized, Baker Chemicals) were used without further purification. 
Table 1. Intrinsic viscosities $[\eta]$ of PPO samples as a function of molecular weight and polydispersity. Temperature $25^{\circ} \mathrm{C}$

\begin{tabular}{cccccc}
\hline PPO* & $\overline{\mathbf{M}}_{\mathbf{w}}$ & $\overline{\mathbf{M}}_{\mathbf{w}} / \overline{\mathbf{M}}_{\mathbf{n}}$ & $\overline{\mathbf{M}}_{\mathbf{z}} / \overline{\mathbf{M}}_{\mathbf{w}}$ & $\begin{array}{c}{[\eta]} \\
(\mathrm{ml} / \mathrm{g}) \\
\text { Toluene }\end{array}$ & $\begin{array}{c}{[\eta]} \\
(\mathrm{ml} / \mathrm{g}) \\
\text { Trichloroethene }\end{array}$ \\
\hline 1 & $8000 \dagger$ & $1.6 \dagger$ & $1.4 \dagger$ & - & $16.2 \ddagger$ \\
2 & 25,000 & 1.6 & 1.4 & $34.2 \ddagger$ & 34.6 \\
3 & 37,000 & 1.3 & 1.4 & 47.8 & 48.2 \\
4 & 39,000 & 1.9 & 1.7 & 48.0 & 45.0 \\
5 & 40,000 & 1.4 & 1.4 & 52.8 & 53.1 \\
6 & 57,000 & 1.4 & 1.4 & 66.6 & 67.9 \\
7 & 63,000 & 1.4 & 1.5 & 70.1 & 70.5 \\
8 & 66,000 & 1.7 & 1.4 & 64.6 & 65.1 \\
9 & 87,000 & 1.9 & 1.2 & 77.5 & 77.9 \\
\hline
\end{tabular}

* Poly(2,6-dimethyl-1,4-phenylene oxide).

$\uparrow$ Reproducibility of mol wts $3-5 \%$.

$\ddagger$ Reproducibility of intrinsic viscosities $0.3-0.6(\mathrm{ml} / \mathrm{g}$ ).

Solutions of the desired concentrations were prepared and stored at room temperature as well as at $60^{\circ} \mathrm{C}$. Measurements at various temperatures were performed by heating as well as by cooling the solutions to the desired temperature.

\section{Light scattering measurements}

We performed light scattering measurements at 4-5 concentrations below $1.5 \%(\mathrm{w} / \mathrm{v})$ and at 3 concentrations above $1.5 \%$ in a Fica 50 light scattering photometer, using vertically polarized light of wavelength $546 \mathrm{~nm}$. This wavelength is too high for the radii of gyration $\left(R_{g z}\right)$ we expected; $\mathbf{R}_{\mathrm{gz}}$ should exceed $\lambda^{\prime} / 20$ (where $\lambda^{\prime}$ is the wavelength in the solution.) In our case $R_{\mathrm{gz}}$ just was of the order of $\lambda^{\prime} / 20$. We had to resort to this wavelength because our solutions always had a brownish colour (high mol wt were even strongly coloured) and absorbed light at 436 and $365 \mathrm{~nm}$.

Reproducible determinations of $\overline{\mathbf{M}}_{w}$, second virial coefficient and radius of gyration were more easily achieved for the toluene solutions than for the TCE solutions. The best results for the latter were obtained for solutions clarified by filtering through Fluoropore $0.2 \mu$ filters (of Millipore Corp.); for the toluene solutions, the conventional Millipore $0.22 \mu$ could be used. Light scattering measurements were performed at 11 angles between 30 and $150^{\circ}$ to the incident beam. For the calibration constants and an example of a light scattering plot, see Koenhen and Smolders [1].

Refractive index increments were measured on a Brice Phoenix refractometer. We obtained $\mathrm{d} n / \mathrm{d} c$ values at 25,35 , 40 and $60^{\circ}$ of $0.116,0.128,0.133$ and 0.136 for toluene solutions, and $0.139,0.145,0.152$ and 0.160 for TCE solutions.

\section{Viscometry}

A capillary viscometer of the Ubbelohde suspendedmeniscus type, with efflux time of $135 \mathrm{sec}$ for toluene and $60 \mathrm{sec}$ for TCE at $25^{\circ} \mathrm{C}$, was used. For the determination at higher temperatures, the viscometer was closed with a silicon-rubber stopper between the measurements to reduce evaporation of the solvent. Relative viscosities $\eta_{r}$ were calculated directly as $t / t_{0}, t$ being the efflux time for the solution and $t_{0}$ that for the solvent. We determined $\eta_{r}$ for 2 series of measurements per PPO-sample or per temperature, each series consisting of 5-6 concentrations in the range $(1-10) \times 10^{-3} \mathrm{~g} / \mathrm{ml}$.

For each series, $\left(\ln \eta_{r}\right) / c$ and $\left(\eta_{r}-1\right) / c$ were plotted against concentration $c$. Always straight lines were obtained, with regression coefficients better than -0.96 and 0.99 , respectively. The intrinsic viscosity $[\eta]$ was calcu- lated as the mean value of the intercepts. In spite of the good correlation, the slopes of the Huggins plot [13] $\left(\eta_{r}-1\right) / c$ vs $c$ did not reproduce very well: Huggins' coefficients $k_{H}$ in the range $0.42-0.49$ were calculated (for both solvents).

The values of $[\eta]$, presented in the tables and in the graph, are the mean values, obtained in the way just described for the 2 series of measurements per temperature or per PPO-sample.

\section{Osmometry}

A Hallikainen Model 1361 automatic osmometer was used with gel cellophane membranes (Sartorius membrane filter SM 11539). Measurements at 5 concentrations below $1.5 \%$ were carried out in toluene as a solvent.

\section{Gel-permeation chromatography}

A Waters gel-permeation chromatograph was used with a series arrangement of four columns $\left(\frac{3}{8}\right.$ in. $\times 4 \mathrm{ft}$ each) packed with styragels of porosity ratings $10^{5}, 3 \cdot 10^{4}, 10^{3}$ and $250 \AA$. Tetrahydrofuran was used as solvent with a column temperature of $30^{\circ} \mathrm{C}$. The flow rate was $1 \mathrm{ml} / \mathrm{min}$. A differential refractometer with a flowing reference cell was used as detector. The columns were calibrated with PPO-samples of known $\overline{\mathbf{M}}_{n}$ - and $\overrightarrow{\mathbf{M}}_{w}$-values, previously determined by osmometry and light scattering. An empirical peak-broadening correction was applied, based on GPC measurements on polystyrenes for which $\overline{\mathbf{M}}_{n}, \overline{\mathbf{M}}_{w}$ and $\overline{\mathbf{M}}_{z}$ were known (osmometry, light scattering, equilibrium distribution in the ultracentrifuge (cf. Ref. [14]).

\section{RESULTS}

\section{Characteristic parameters of $P P O$}

Our PPO samples generally had lower mol wts than those studied earlier $[10,11]$. Our intrinsic viscosities were 5-15 units higher than those of others $[10,11]$ for comparable mol wts. We ascribe this to differences in polydispersity between the samples. In order to take polydispersity into account, we inserted values of $\overline{\mathbf{M}}_{w}$ in all the formulae, mentioned in this paper.

We determined for all samples by means of a least squares analysis the parameters $K_{\mathrm{w}}$ and $a$ of the equation

$$
[\eta]=K_{\mathrm{w}} \overline{\mathbf{M}}_{\mathrm{w}}^{a}
$$

and found for toluene at $25^{\circ} \mathrm{C}$

$K_{\mathrm{w}}=(3.1 \pm 0.2) \times 10^{-3}$ and $a=0.70 \pm 0.01$ 
and for TCE at $25^{\circ} \mathrm{C}$

$K_{\mathrm{w}}=(3.3 \pm 0.2) \times 10^{-3}$ and $a=0.70 \pm 0.01$.

These values differ slightly from those previously published $[10,11]$. This may be ascribed not only to differences in polydispersity, but also to the smaller molecular weight region studied by us.

From the Stockmayer-Fixman equation, as modified by Yamakawa [15]

$$
[\eta] / \overline{\mathrm{M}}_{\mathrm{w}}^{0.5}=K_{\theta}+0.346 \Phi_{0} B \overline{\mathrm{M}}_{\mathrm{w}}^{0.5}
$$

we calculated, with the data for all our PPO samples, the unperturbed dimension parameter $K_{0}$ and the solvent-solute interaction ("excluded volume") parameter $B$. For $\Phi_{0}$ we used the value $2.5 \times 10^{23}$ [15]. For toluene we found $K_{0}=0.170 \pm 0.010$ and $B=$ $(2.2 \pm 0.4) \times 10^{-27}$ and for TCE $K_{0}=0.155 \pm 0.015$ and $B=(2.5 \pm 1.0) \times 10^{-27}$. These values again differ slightly from those of other workers $[10,11]$ for comparable solvents.

The above values for $K_{0}$ and $B$ were calculated (by means of least-squares analyses) for $25^{\circ} \mathrm{C}$. Unfortunately, the points were too scattered to enable us to find a trend in $K_{\theta}$ and/or $B$ with raising temperature: all $K_{t}$ and $B$ values, determined at higher temperatures were within experimental error equal to those at $25^{\circ} \mathrm{C}$.

From the $K_{\theta}$ we calculated $[\eta]_{0}$ and from $[\eta]_{\theta}$ and $[\eta]$ we calculated expansion factors $\alpha_{\eta}[15]$. We found $\alpha_{\eta}$ in the range $1.1-1.2$, in good agreement with earlier values $[10,11]$. All the values of $\alpha_{n}$ at all temperatures were found to be in this range. Thus, $\alpha_{1}$ for PPO is not only small as compared to other coil polymers [11] but also is nearly independent of temperature.

Our value of $\alpha_{n}$ gives an excluded volume parameter $z$ according to [15]:

$$
\alpha_{\eta}^{3}=1+1.05 z
$$

in the range $0.3-0.7$. The interpenetration factor $\psi$, defined by [15]:

$$
A_{2}=4 \pi^{1.5} N_{\mathrm{Av}}\left(\mathrm{R}_{\mathrm{gw}}^{3} / \overline{\mathrm{M}}_{\mathrm{w}}^{2}\right) \psi
$$

and calculated from the data of Table 2 (with $\mathrm{R}_{\mathrm{gw}}$ approximated by

$$
\mathrm{R}_{\mathrm{gw}}=\left(\left(\overline{\mathrm{M}}_{\mathrm{w}} / \overline{\mathrm{M}}_{\mathrm{z}}\right) \cdot \mathrm{R}_{\mathrm{gz}}\right)
$$

is for PPO in TCE and toluene in the range 0.17-0.30. The agreement with other values of $\psi$, experimentally determined or theoretically derived (cf. [15]), is not good: a $\psi$ value of 0.30 is out of range, even for $z=0.7$. This might mean that the averaged values for $A_{2}$ given in Table 2 are too high. This supposition is confirmed by the calculation of the dimensionless parameters $A_{2} \overline{\mathrm{M}}_{\mathrm{w}} /[\eta]$ : they are also too high (1.2-1.5), as they are expected to be 1.0-1.2 [15].

The values of the radii of gyration were, at all temperatures, in the ranges given in Table 2 . No definite trend in $\mathbf{R}_{\mathrm{g} z}$ with rising temperature was found. The poor reproducibility in the determinations of the radius of gyration especially in TCE solutions implied that we were not able to determine reliable solubility $(\chi-)$ parameters for the TCE solutions from the same measurements by Scholte's method [16]. This method was applied to the PPO-toluene system by Koenhen et al. [1]; within experimental error, we found for this
Table 2. Radius of gyration (z-average) and second virial coefficient $A_{2}$ as determined from light scattering. Temperature $25^{\circ} \mathrm{C}$

\begin{tabular}{ccccc}
\hline PPO & $\overline{\mathrm{M}}_{w}$ & Solvent & $\begin{array}{c}\mathrm{R}_{\mathrm{gz}} \\
(\AA)\end{array}$ & $\begin{array}{c}A_{2} \times 10^{4} \\
\left(\mathrm{~mol} \cdot \mathrm{ml} / \mathrm{g}^{2}\right)\end{array}$ \\
\hline 8 & \multirow{2}{*}{66,000} & TCE* $^{*}$ & $160 \pm 30$ & $12 \pm 6$ \\
& & Tolt & $140 \pm 20$ & $15 \pm 3$ \\
9 & \multirow{2}{*}{87,000} & TCE & $180 \pm 30$ & $10 \pm 2$ \\
& & Tol & $160 \pm 20$ & $10 \pm 2$ \\
\hline
\end{tabular}

* Trichloroethene.

$\dagger$ Toluene.

system the same $\chi$-parameters and the same dependence of $\chi$ on concentration as Koenhen et al. We confirm their finding that $\chi$ is independent of temperature, within experimental error. For PPO-TCE nearly the same values were found for $A_{2}$ and $\mathrm{R}_{\mathrm{gz}}$ as for PPO-toluene; we conclude that the $\chi$-parameters for PPO-TCE do not differ appreciably from those for PPO-toluene, in the dilute solution range. Thus, in this range TCE should not be regarded as a "better" solvent for PPO than toluene.

\section{Intrinsic viscosity as a function of temperature}

The results of our viscosity measurements (Fig. 1) indicate a conformational transition. The $[\eta]-T$ curves show a point of inflection in the range $45-35^{\circ}$; the higher the mol wt, the more pronounced is the effect. Such a dependence on molecular weight for the effects of a conformational transition has been found earlier for other polymers [7].

The intrinsic viscosity depends (a) on the solvent draining (or the permeability) of the macromolecules; (b) on the skeletal geometry; and (c) on the excluded volume $(B$ or $z$ ) [the unperturbed dimension parameter $K_{t}$, derived e.g. from Eqn (2), depends on both (a) and (b)]. Hence, determination of the temperature dependence of one of these three parameters from a measured [ $\eta]-T$ curve (cf. Ref. [9]) presupposes knowledge (experimental and/or theoretical) concerning the temperature dependence of the other two parameters.

Evans et al. [8] showed that a maximum in the $[\eta]-T$ curve, or a steady decrease with increasing $T$, always means that the temperature coefficient of the unperturbed dimension $\left(\mathrm{d} \ln K_{\theta} / \mathrm{d} T\right)$ is negative, irrespective of the assumptions made about the dependence of $[\eta]$ on $\alpha_{\eta}$ and about the dependence of $\alpha_{\eta}$ on the excluded volume parameter $z$. The analysis of Evans et al. [8] is based however on the assumption that $z$ increases with increasing $T$, which means that $\alpha_{\eta}$ should not decrease with increasing $T$.

We found experimentally that the expansion factor $\alpha_{\eta}$ is, within experimental error, independent of temperature. We may therefore conclude that, for PPOTCE and for PPO-toluene, $\mathrm{d} \ln K_{t} / \mathrm{d} T$ is negative. This confirms the earlier, comparable, conclusion of Akers et al. [11] based on Stockmayer-Fixman plots (at 25 and $90^{\circ} \mathrm{C}$ only, however). Thus, reasoning along the lines pointed out by Evans et al. [8], we arrived at a qualitative conclusion about the behaviour of $K_{\theta}$ as a function of temperature; from our Stockmayer-Fixman plots, we could not reach such a conclusion. In 


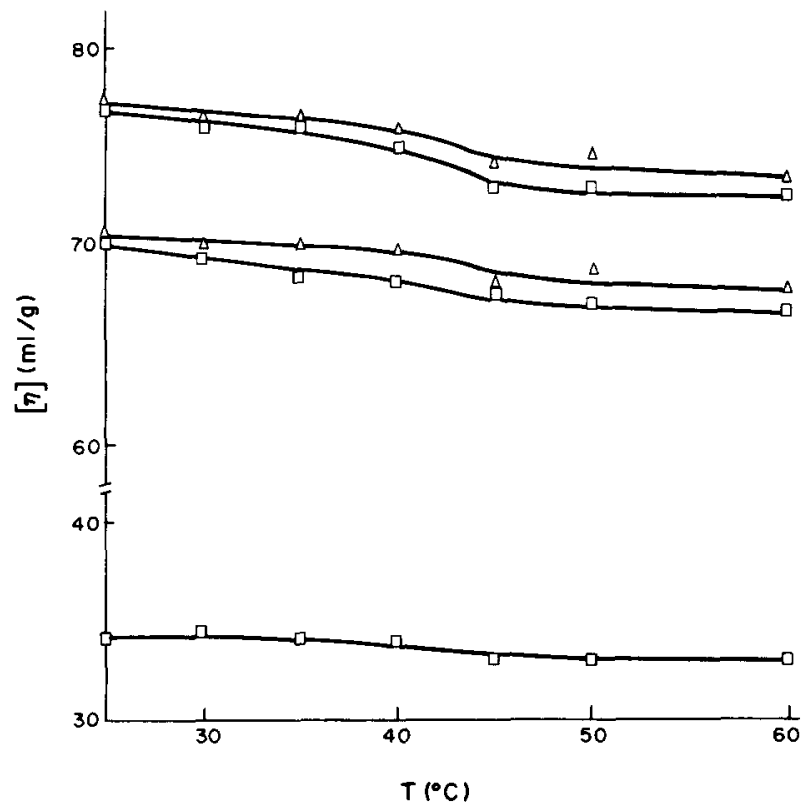

Fig. 1. Intrinsic viscosity as a function of temperature, for PPO samples of three molecular weights. Upper two curves $\overline{\mathrm{M}}_{\mathrm{w}}=87,000$, middle two curves $\overline{\mathrm{M}}_{\mathrm{w}}=63,000$, lowest curve $\overline{\mathrm{M}}_{\mathrm{w}}=25,000$. $\Delta$ Solutions in TCE; $\square$ solutions in toluene.

our opinion, the conclusion that $\mathrm{d} \ln K_{\theta} / \mathrm{d} T<0$ holds for the left part of the $[\eta]-T$ curve (i.e. to the left of the point of inflection) as well as for the right part.

Thus, assuming that the permeability is independent of $T$, we conclude that the peculiar shape of the $[\eta]-T$ curve means that the tightly coiled dissolved PPO molecules adopt a slightly less coiled, somewhat more expanded structure when the solution is lowered in temperature. We believe that the existence of a point of inflection means that this dimensional change is more abrupt than gradual.

The conformational transition occurs in the same temperature region for TCE and toluene. Thus, our hypothesis (that different temperature regions for a conformational transition, if present, should be found) is not confirmed.

Change of characteristic parameter $\mathrm{C}_{\infty}$ with temperature

In order to give a more quantitative interpretation to our measurements of $[\eta]$ at different temperatures, we calculated characteristic ratios $C_{\infty}$ from the intrinsic viscosities.

The characteristic ratio $C_{\infty}$ is defined as [17]:

$$
C_{\infty}=\lim _{\overline{\mathrm{N}}_{\mathrm{w}} \rightarrow \infty} \frac{6 \mathrm{R}_{\mathrm{gw}}^{2} / \alpha_{\mathrm{s}}^{2}}{\overline{\mathrm{N}}_{\mathrm{w}} l^{2}} \approx \frac{6 \mathrm{R}_{\mathrm{gw}}^{2} / \alpha_{\eta}^{2}}{\overline{\mathrm{N}}_{\mathrm{w}} l^{2}}
$$

where

$\alpha_{\mathrm{s}}=$ expansion factor for radius of gyration $\left(\alpha_{\mathrm{s}} \neq \alpha_{\eta}\right)$

$\overline{\mathbf{N}}_{\mathrm{w}}=$ weight-average degree of polymerization $\left(\mathbf{N}_{\mathrm{w}}\right.$ is about 700 in our case, and considered to be "large enough")

$l=$ bond length of repeating unit $(1=5.4 \AA$ $[10,11])$.

The ratio $C_{\infty}$ is related to $K_{\theta}$ by

$$
C_{\infty}=\left(\frac{K_{\theta}}{\Phi_{0}}\right)^{2 / 3} \frac{m}{l^{2}}
$$

where

$$
m=\text { molecular weight of repeating unit ( } m=120 \text { ). }
$$

The use of the parameter $C_{\infty}$ has no special advantages over $K_{\theta}$, except that it has been the subject of numerous theoretical calculations [17] for many types of polymer chains (also as a function of temperature). In order to calculate $C_{\infty}$, we first calculated $\mathbf{R}_{\mathbf{g w}}$ from the measured intrinsic viscosities. To this end we assumed the PPO molecules to be in the non-draining limit. We justify this assumption by referring to the paper of BarralesRienda and Pepper [10], who called the PPO molecules "effectively impermeable". (We could confirm their conclusion by calculating permeabilities from sedimentation and flotation coefficients, obtained from experiments with the analytical ultracentrifuge.) For this model, the Flory-Fox formula [15] relates $[\eta]$ to $\mathbf{R}_{\mathrm{gw}}$ :

$$
[\eta]=6^{3 / 2} \Phi \frac{\left(\mathrm{R}_{\mathrm{gw}}\right)^{3}}{\overline{\mathrm{M}}_{\mathrm{w}}}
$$

where

$$
\Phi=\Phi_{0}(1-0.46 z)
$$

Another version of the Flory-Fox formula reads [15]:

$$
[\eta]=6^{3 / 2} \Phi_{0} \frac{\left(\mathbf{R}_{\mathrm{gw}}\right)_{0}^{3} \alpha_{\eta}^{3}}{\overline{\mathrm{M}}_{\mathrm{w}}}
$$

where $\left(\mathbf{R}_{\mathbf{g w}}\right)_{0}=$ unperturbed radius of gyration, from which $C_{\infty}$ is calculated as:

$$
C_{\infty}=\frac{6\left(\mathrm{R}_{\mathrm{gw}}\right)_{0}^{2}}{\overline{\mathrm{N}}_{\mathrm{w}} l^{2}} \text {. }
$$

Assuming that $\alpha_{\mathrm{s}}=\alpha_{\eta}=1.1$, for all four temperatures, we calculated $R_{\mathrm{gw}},\left(\mathrm{R}_{\mathrm{gw}}\right)_{0}$ and $C_{\infty}$ from Eqns (5) and (9) as shown in Table 3

Calculation of $K_{\theta}$ from $C_{\infty}$ by means of Eqn (6) leads to values within experimental error equal to 
Table 3. Radius of gyration (weight average) and characteristic ratio $C_{\alpha}$ as a function of temperature, calculated from intrinsic viscosities by means of the Flory-Fox equation. Molecular weight $\overline{\mathrm{M}}_{\mathrm{w}}$ of PPO: 87,000

\begin{tabular}{ccccccc}
\hline $\begin{array}{c}\text { Temp. } \\
(\mathrm{C})\end{array}$ & Solvent & $\begin{array}{c}{[\eta]} \\
(\mathrm{ml} / \mathrm{g})\end{array}$ & $\begin{array}{c}\mathrm{R}_{\mathrm{gw}} \\
(\AA)\end{array}$ & $C_{x}$ & $\begin{array}{c}\left(\mathrm{R}_{\mathrm{gw}}\right)_{0} \\
(\AA)\end{array}$ & $C_{x}$ \\
\hline 25 & TCE & 77.9 & $129^{*}$ & $4.0 \dagger$ & $112 \ddagger$ & $3.6 \$$ \\
35 & TCE & 76.5 & 128 & 3.9 & 111 & 3.6 \\
45 & TCE & 74.1 & 127 & 3.9 & 110 & 3.5 \\
60 & TCE & 73.5 & 126 & 3.8 & 109 & 3.5 \\
25 & Tol & 77.5 & 129 & 4.0 & 111 & 3.6 \\
35 & Tol & 76.0 & 128 & 3.9 & 111 & 3.6 \\
45 & Tol & 73.0 & 126 & 3.8 & 109 & 3.5 \\
60 & Tol & 72.0 & 125 & 3.7 & 109 & 3.5 \\
\hline
\end{tabular}

* Calculated from Eqn (7) with $z=0.3$.

+ Eqn (5) with $a_{v}=\alpha_{11}=1.1$.

$\ddagger$ Eqn (8) with $\alpha_{n}=1.1$.

$\$$ Eqn (9).

those derived from Stockmayer-Fixman plots. Thus the relative change in $C_{\infty}$ with temperature, calculated from the use of the Flory-Fox formula and the assumptions about $\alpha_{17}$, is compatible with (though not confirmed by) the results of the Stockmayer-Fixman plots.

\section{DISCUSSION}

In toluene as well as in trichloroethene, we found PPO to exhibit a conformational transition in the range $45-35^{\circ} \mathrm{C}$, from a tightly coiled structure at temperatures above $45^{\circ} \mathrm{C}$ to a more expanded form below $35^{\circ} \mathrm{C}$. We found no differences between toluene and TCE as to the temperature range where the transition occurs.

For other polymer-solvent systems, dramatic conformational transitions with large changes in $[\eta]$ or in $R_{\mathrm{gz}}$ have been found [5-7]. The conformational transition discovered for PPO is of a more modest type. As we gave the solutions sufficient time to reach temperature equilibrium, and as we approached the desired temperatures from above as well as from below, we may consider the conformational transition of PPO to be real, and not to be an artefact caused by kinetic effects.

That the PPO molecules expand when the temperature is lowered can be explained by assuming that in solution those helices are formed which occur in the crystals [3]. We cannot support the explanation by referring to the results of conformational calculations for PPO. As far as we are aware, no such calculations have been carried out. Assuming, however, that the PPO chain in its segment mobility behaves like the poly(oxymethylene) chain (for which calculations of the conformation as a function of helix content and temperature exist [17]), we think that it is justified to say that lowering of temperature increases the number of helical sequences as well as the unperturbed dimensions.

An explanation for the relatively small change in unperturbed dimensions with temperature may be that the potential barriers to rotations or crankshaft movements of (groups of) monomer units are rather high with respect to $R T$. They are of the order of $5 \mathrm{~kJ}$ for rotations and of $25 \mathrm{~kJ}$ for crankshaft movements [18], whereas $R T$ is about $2.5 \mathrm{~kJ}$.

Strictly speaking, we found the conformational transition only for infinitely dilute solutions. The radius of gyration, however, depends on concentration [15]. Thus, we might doubt that a conformational transition (i.e. a change in radius of gyration with temperature) found for infinitely dilute solutions would persist in more concentrated solutions. Theories for dilute solutions [15] (up to $2-3 \%$ polymer, in our case), where no extensive overlapping of polymer chains occurs, indicate that the way in which the radii of gyration change with concentration should be the same for each temperature, provided that the excluded volume parameters $z$ and $B$ may be assumed to be independent of temperature.

Assuming this, we may then infer that for dilute PPO solutions (up to $2-3 \%$ ) the same conformational transition occurs as that found for infinitely dilute systems. Toluene solutions containing $2-3 \%$ PPO crystallize in the temperature region $8-20 \mathrm{C}$. Thus, we may consider as confirmed the hypothesis of Koenhen et al. [2] that nucleation and growth of crystallites is preceded by a conformational transition, at least for dilute toluene solutions up to $2-3 \%$ PPO.

Acknowledgement-We thank Professor J. Schuyer for reading the manuscript and for his helpful comments.

\section{REFERENCES}

1. D. M. Koenhen and C. A. Smolders, J. Polym. Sci Polym. Phys. Ed. 15, 155, 167 (1977).

2. D. M. Koenhen, C. A. Smolders and M. Gordon, $J$. Polym. Sci. Polym. Symp. 61, 93 (1978).

3. E. Turska and H. Janeczek, Polymer 19, 81, 85 (1978).

4. L. Broens, D. M. Koenhen and C. A. Smolders, Desalination 22, 205 (1977).

5. A. Dondos and H. Benoit, C. r. Acad. Sci. Paris 271, 1055 (1970).

6. I. Katime and C. Ramiro Vera, Eur. Polym. J. 13, 451 , 783 (1977).

7. A. Teramoto, K. Nagakawa and H. Fujita, J. chem. Phys. 46, 4197 (1967)

8. J. M. Evans, M. B. Huglin and R. F. T. Stepto, Makromolek. Chem. 146, 91 (1971).

9. H. Suzuki, Y. Miyazaki and K. Kamide, Eur. Polym. J 16, $703(1980)$.

10. J. M. Barrales-Rienda and D. C. Pepper, Eur. Polym. J. 3, 535 (1967).

11. P. J. Akers, G. Allen and M. J. Bethel, Polymer 9, 575 (1968).

12. E. M. McCaffery, Laboratory Preparation for Macromolecular Chemistry. McGraw-Hill, New York (1970).

13. B. A. Wolf, In Polymer Handbook (Edited by J. Brandrup and $E$. $H$. Immergut), 2nd Edition, pp IV-135/136. Wiley, New York (1975).

14. Th. G. Scholte, J. Polym. Sci. A-2 6, 91, 111 (1968).

15. H. Yamakawa, Modern Theory of Polymer Solutions, pp. $168,169,214,215.300,301,314,364.365 .374,375$, 384. Harper \& Row, New York (1971).

16. Th. G. Scholte, Eur. Polym. J. 6, 1063 (1970).

17. P. J. Flory, Statistical Mechanics of Chain Molecules, pp. 11, 33, 36-38, 159-165. Wiley Interscience, New York (1969)

18. A. A. Jones and R. P. Lubianez, Macromolecules 11, $126(1978)$ 